\title{
Turbulence: Numerical Analysis, Modeling, and Simulation
}

\author{
William Layton \\ Department of Mathematics, University of Pittsburgh, Pittsburgh, PA 15260, USA; wj1@pitt.edu
}

Received: 13 February 2018; Accepted: 14 February 2018; Published: 18 February 2018

The problem of accurate and reliable prediction of turbulent flows is a central and intractable challenge that crosses disciplinary boundaries. As the needs for accuracy increase and the applications expand beyond flows where extensive data is available for calibration, the importance of a sound mathematical foundation that addresses the needs of practical computing increases. This special issue is directed at this crossroads of rigorous numerical analysis, the physics of turbulence, and the practical needs of turbulent flow simulations. It contains papers providing a broad understanding of the status of the problem considered and open problems that comprise further steps. It consists of papers covering fundamentals, applications, theory, simulations, experiments, and reviews. The papers cover the general topics summarized below.

Kubacki and Tran [1] present a modern, efficient approach for uncoupling groundwater-surface water flows governed by the fully evolutionary Stokes-Darcy equations. These algorithms treat the coupling terms explicitly and at each time level require only one sub-physics, sub-domain solve that can be done by codes highly optimized for individual processes. Obviously, such methods have greater accuracy and efficiency per time step than non-optimized, fully coupled, monolithic methods. Thus, the key to their utility is whether a price in stability must be paid. This paper presents algorithms with unconditional stability and high accuracy.

Nguyen et al. [2] study the simulation and modeling of the dispersion from an instantaneous source of heat or mass located at the center of a turbulent flow channel. This work is at the intersection of high impact in applications and the leading edge of the understanding of turbulence modulation by transport effects.

Bowers and Rebholz [3] present a review of recent results for the reduced Navier-Stokes- $\alpha$ (rNS- $\alpha)$ model of incompressible flow. The model was recently developed as a numerical approximation of the well-known Navier-Stokes- $\alpha$ model. Numerical simulations are far more efficient with the reduced model. Those simulations have revealed interesting features of the reduced model as an independent fluids model.

Basse [4] presents a comparison of turbulence intensity profiles for smooth and rough wall pipe flow measurements made in the Princeton Superpipe. The profile development in the transition from smooth to rough wall flow is analyzed from the data. In this paper, the highly difficult problem wherein maximum insight must be obtained from available data is addressed.

Chen and Lo [5] present a numerical study of coherent structure evolution in boundary layer transition flow using high order compact difference schemes with non-uniform grids in the wall-normal direction. Efficient solutions and high accuracy are provided in this interesting study.

Maulik and San [6] present the results of a study solving two-dimensional (2D), compressible turbulence. Their paper compares two promising computational approaches and draws valuable conclusions.

Brkić and Ćojbašić [7] present evolutionary optimization for approximations of the Colebrook's equation for the turbulent friction factor. This calculation is used for the calculation of turbulent hydraulic resistance in hydraulically smooth and rough pipes including the transient zone. 
Breckling et al. [8] present an overview of time relaxation models. To date, these have been one of the few models for LES where the model solution is proven to converge to the true averages of the turbulent flow. This approach is related to data assimilation by a technique called nudging. It has proven to be effective in the regularization of Navier-Stokes equations, and as such this summary of completed theory, necessary algorithms, and new directions is very welcome.

Dunca [9] studies a very promising family of alpha-deconvolution models. It is widely observed that high-order models and methods always outperform low-order, ones even for problems with rough solutions when the available theory indicates no advantage. This paper is one of the first theoretical studies to explain this advantage. The paper provides a theoretical analysis of model accuracy with many complicating factors that would suggest no advantage of high-order models to compensate for their greater cost. This paper is a landmark theoretical result.

In the final paper [10], Ries et al. present a database generated by numerical and physical experiments of a database of near-wall turbulence properties of a highly turbulent jet impinging on a solid surface under different inclination angles. The dilemma of resolving near-wall turbulence or employing error-prone near-wall models is inescapable in LES. Their database will be useful, even essential, for the development of accurate near-wall models in large-eddy simulations (LES).

I thank all of the contributors for submitting high quality papers for the special issue. I also thank the reviewers for their dedicated time and help supporting the quality of the papers.

Conflicts of Interest: The authors declare no conflicts of interests.

\section{References}

1. Kubacki, M.; Tran, H. Non-Iterative Partitioned Methods for Uncoupling Evolutionary Groundwater-Surface Water Flows. Fluids 2017, 2, 47. [CrossRef]

2. Nguyen, Q.; Feher, S.E.; Papavassiliou, D.V. Lagrangian Modeling of Turbulent Dispersion from Instantaneous Point Sources at the Center of a Turbulent Flow Channel. Fluids 2017, 2, 46. [CrossRef]

3. Bowers, A.L.; Rebholz, L.G. The Reduced NS- $\alpha$ Model for Incompressible Flow: A Review of Recent Progress. Fluids 2017, 2, 38. [CrossRef]

4. Basse, N.T. Turbulence Intensity and the Friction Factor for Smooth- and Rough-Wall Pipe Flow. Fluids 2017, 2, 30. [CrossRef]

5. Chen, W.; Lo, E.Y. High Wavenumber Coherent Structures in Low Re APG-Boundary-Layer Transition Flow-A Numerical Study. Fluids 2017, 2, 21. [CrossRef]

6. Maulik, R.; San, O. Energy Dissipation Characteristics of Implicit LES and Explicit Filtering Models for Compressible Turbulence. Fluids 2017, 2, 14. [CrossRef]

7. Brkić, D.; Ćojbašić, Z. Evolutionary Optimization of Colebrook's Turbulent Flow Friction Approximations. Fluids 2017, 2, 15. [CrossRef]

8. Breckling, S.; Neda, M.; Hill, T. A Review of Time Relaxation Methods. Fluids 2017, 2, 40. [CrossRef]

9. Dunca, A.A. Improving Accuracy in $\alpha$-Models of Turbulence through Approximate Deconvolution. Fluids 2017, 2, 58. [CrossRef]

10. Ries, F.; Li, Y.; Rißmann, M.; Klingenberg, D.; Nishad, K.; Böhm, B.; Dreizler, A.; Janicka, J.; Sadiki, A. Database of Near-Wall Turbulent Flow Properties of a Jet Impinging on a Solid Surface under Different Inclination Angles. Fluids 2018, 3, 5. [CrossRef]

(C) 2018 by the author. Licensee MDPI, Basel, Switzerland. This article is an open access article distributed under the terms and conditions of the Creative Commons Attribution (CC BY) license (http://creativecommons.org/licenses/by/4.0/). 